\title{
Being smarter with smartphones
}

$\mathrm{S}$ eemingly overnight, smartphones have become the latest must-have, irreplaceable tool in the armamentarium of the contemporary clinician. However, their use is often banned or seriously limited in health care institutions, best depicted by ominous signage from Big Brother - "no cellphones or electronic devices may be used on hospital property."

Despite the bans, it is nearly impossible to ignore the "click" of a smartphone being pulled from holsters on rounds. Students, trainees and early adopters among us have embraced the notion that these devices improve clinical care because of rapid access to essential point-of-care information, including laboratory work, reference texts, recent research and up-todate guidelines. As they say, "there's an 'app' for that" - all implicit with the use of these apps is the hope of facilitating rapid bedside decisions and improving care.

As compared with pagers, smartphones can improve communication by minimizing disruptions, because clinicians are able to prioritize nonurgent communication, while also providing instant communication between health care team members. ${ }^{1}$ Treatment and learning may also be facilitated by capturing images of interesting rashes or diagnostic images, or even recording videos of procedures to share with others.

The major unintended consequence of the proliferation of personal smartphones has to do with patient confidentiality. Individuals on the health care team may purposely use smartphones to breach confidentiality, by posting patient information, images or both to social networking sites. For example, in Los Angeles, California, four health care workers were fired and three reprimanded after it was found they used their smartphones to post pictures of a dying patient on Facebook. ${ }^{2}$ Physicians also routinely answer their phones and discuss confidential patient information in environments that they previously would have avoided for such discussions - cafeterias, elevators and other public places inside the hospital or out - forgetting that sensitive information is being projected in very public settings.

Disruptive communications or distractions from clinical duties have also been identified as a concern. For example, one would be hard-pressed to find a health care team in which at least one member is not engaged on their smartphone during rounds. While you attend to the flashing light or fancy ring tone, others are usually wondering if you are looking up a life-saving dose of an inotrope, answering a text regarding dinner plans or simply being rude. Although technologies themselves may be neutral, their use or misuse may not be.

How then might we maximize the potential of this new technology while minimizing its negative impact? First, let's repeal policies banning cellphones in acute and chronic care institutions. Clearly, they are dated and ineffective. Although minor safety risks attributed to electromagnetic interference do exist, they are generally overblown and inappropriately used to justify outright bans.
A more rational approach to regulation of smartphones is needed. For example, limiting use near monitoring equipment in patient rooms in emergency and critical care areas, where evidence has shown they may interfere if in proximity to certain equipment. ${ }^{3-5}$

Once the bans have been lifted, hospitals must develop systems to engage staff in assuring appropriate guidance for use. Although many such guidelines exist, institutional regulations should elaborate on new potential pitfalls in patient confidentiality, usage of devices near electronic equipment, communication protocols and smartphone etiquette aimed at minimizing disruptions (i.e., proper usage on rounds or in group settings).

A number of more specific provisions to ensure confidentiality might be considered. For instance, ensuring that appropriate patient consent is always obtained before any pictures are taken, limiting if not banning the use of social media with respect to potentially identifiable clinical encounters and specifically mandating secure personal passwords on devices in case of loss. Additionally, clinicians should be reminded of the dangers of multi-tasking and distraction in the middle of clinical duties so that they think twice before answering any device. Most hospitals simply need an addendum to existing privacy and electronic communication policies. A 2009 report out of the National Health Service in Britain has also highlighted many of these issues and proffered various potential solutions. ${ }^{6}$

Once established, institution-specific rules should be widely disseminated, through inclusion in all orientation materials as well as clinical department handbooks. For house staff, opportunities to discuss the issues arise at the beginning clerkship for medical students and at hospital orientations for residents, and can be constantly reiterated through signage around hospitals.

It is time to abolish bans on smartphones in health care institutions. More importantly, smart use will ensure that a neutral technology is harnessed to enhance every patient encounter.

Note: The writing of this editorial was interrupted by 3 nonurgent calls, 9 text messages, 24 emails and 1 tweet.

\section{Daniel Rosenfield MD, Paul C. Hébert MD MHSc, Matthew B. Stanbrook MD PhD, Noni E. MacDonald MD MSc, Ken Flegel MDCM MSc}

For references, see Appendix 1, available at www.cmaj.ca/lookup/suppl /doi:10.1503/cmaj.110524/-/DC1.

Competing interests: See www.cmaj.ca/site/misc/cmaj_staff.xhtml. None declared by Daniel Rosenfield.

Affiliations: See www.cmaj.ca/site/misc/cmaj_staff.xhtml. Daniel Rosenfield is from the Faculty of Medicine, University of Toronto, Toronto, Ont.

Correspondence to: CMAJ editor, pubs@cmaj.ca

CMAJ 2011. DOI:10.1503/cmaj.110524

All editorial matter in CMAJ represents the opinions of the authors and not necessarily those of the Canadian Medical Association. 